\title{
Electrical resistivity of holmium hydride films
}

\author{
M S RAHMAN KHAN \\ Department of Applied Physics and Electronics, University of Rajshahi, Rajshahi, \\ Bangladesh \\ MS received 2 June 1986; revised 3 March 1987
}

\begin{abstract}
The electrical resistivity and temperature coefficient of resistance of polycrystalline holmium hydride films, grown on glass slides, have been measured in vacuo. Experimental results show that the resistivity decreases with decreasing film thickness. The resistivity of $200 \AA$ holmium dihydride film is less than that of the parent metal. The observed behaviour of the thickness dependence of the resistivity has been explained in terms of the structural phase change.
\end{abstract}

Keywords. Holmium hydride films; electrical resistivity; temperature coefficient of resistance.

\section{Introduction}

Although considerable work has been done on the electrical properties of thin films of metal (Chopra 1969; Maissel and Glang 1970; Samble 1983), not much is known about the thin films of rare earth metals and their hydrides. It was therefore feit worthwhile to carry out some investigations on the electrical properties of the films grown from holmium which is one of the heavy rare earth metals.

Conductivity measurements have earlier been made on most of the rare earth metals and their hydrides in the bulk form (Heckman 1969; Kubota and Wallace 1963). The results show a wide variation of conductivity of the $\mathrm{Ho}-\mathrm{H}$ system depending upon its composition, e.g. the nonstoichiometric dihydride has higher conductivity than the parent metal.

The electronic properties of rare earth hydrides are usually discussed in terms of either protonic hydrogen model or the anionic hydrogen model (Libowitz 1968; Bos and Gayer 1966; Heckman 1969; Colvin et al 1960). In the former, the hydrogen contributes an electron to the system's conduction band, while in the anionic model it extracts one. It has been shown that when holmium metal (having an h.c.p. structure) is thermally evaporated, the condensed film form f.c.c. dihydrides upto $200 \AA$ thickness (Khan and Miller 1979; Curzon and Chlebeck 1972; Curzon and Singh 1975; Gasgnier et al 1974). Films thicker than $820 \AA$ have h.c.p. bulk structure while films of intermediate thickness have a mixed phase (f.c.c. dihydride + h.c.p. metal). This paper aims at reporting the anomalous behaviour of the resistivity of the film prepared from holmium and to correlate them with the structural studies mentioned above.

\section{Experimental}

Thin films of holmium hydride (thickness range 70-900 $\AA$ ) were prepared by vacuum evaporation of holmium wire $(99.9 \%$ purity, Koch Light, U.K.) onto precleaned soda 
glass microscope slides at a pressure of about $10^{-6}$ torr. The materials were evaporated from helical tungsten filament.

Making reliable electrical contact to the films is always a problem, but this was overcome by constructing 4 spring-loaded flat ended copper rods $(0.15 \mathrm{~cm}$ diameter $)$ fixed with the mask so that they could be easily adjusted to make good electrical contact with the predeposited nichrome electrode on the substrate. The system was found to give a satisfactory ohmic contact to the film in the temperature range $77^{\circ} \mathrm{K}$ to $573^{\circ} \mathrm{K}$. By using suitable masks, nichrome films of about $1500 \AA$ thickness were deposited on the previously cleaned substrates to form contact electrodes and were found very effective. Contacts to the films were made by evaporating the holmium hydride films over the predeposited nichrome electrodes.

The resistance measurement in this work was carried out employing a conventional four-probe technique (Vander Pauw 1958). A constant current in the range $200-500 \mu \mathrm{A}$ was passed through the specimen between two probes and the voltage developed between the other two probes was measured. The voltage across the two potential probes was measured using a d.c. microvoltmeter sensitive to $100 \mu \mathrm{V}$. The current was measured with an electrometer sensitive to $10^{-14}$ amps. The resistivity value for a film of a particular thickness was reproducible, when the observations were repeated on the films of the same thickness grown under identical conditions. The film temperature was varied by using a specially designed radiant heater and was measured by a $T_{1}-T_{2}$ chromel alumel thermocouple. The temperature between the ambient and the liquid nitrogen was achieved by using a conduction cooled cryostat, a very simple device constructed in the laboratory. The film thickness was determined by Tolansky's multiple beam interferometric method.

\section{Results and discussions}

The electrical resistivity (at $77^{\circ} \mathrm{K}$ and in the range $293-573^{\circ} \mathrm{K}$ ) and the temperature coefficient of resistance of the polycrystalline films grown from holmium are discussed here. The structure of the same films has been determined by electron diffraction studies (Khan and Miller 1979) and attempts have been made to correlate the dependence of the electrical resistivity and structures on thickness.

The thickness dependence of the electrical resistivity of holmium hydride films at room temperature is shown in figure 1 . The resistivity of the films thinner than $70 \AA$ could not be accurately measured because of their discontinuous structures (Khan 1976). The curve of figure 1 is reproducible to within experimental error. The nonstoichiometric characteristics of the rare earth hydride, especially dihydride and the lack of knowledge of the exact composition (i.e. $\mathrm{H} / \mathrm{Ho}$ atomic ratio) of the film made it difficult to precisely explain the behaviour of resistivity vs thickness curve. However, attempts have been made to put forward arguments and evidences to explain the characteristics on the basis of bulk dihydride resistivity, our own structural investigations and the observed resistivity values.

An examination of the curve shows its anomalous behaviour. The salient features of the resistivity thickness curve are (i) the resistivity has a minimum value of $60 \mu \Omega-\mathrm{cm}$ at a film thickness of about $200 \AA$ and increases for thinner films and (ii) an

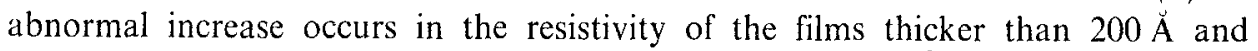
continues to increase until it becomes nearly constant at $900 \AA$. 


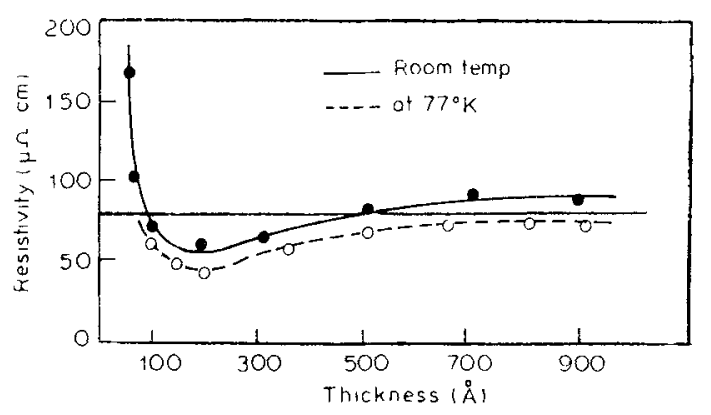

Figure 1. Thickness dependence of resistivity of holmium hydride films (70-900 $\AA$ ).

An interestirig feature of the experimental results is that the resistivity of $200 \AA$ film $(60 \mu \Omega-\mathrm{cm}$ ) is less than that of the parent metal (for bulk Ho. $\rho=81 \mu \Omega-\mathrm{cm}$ ). This clearly indicates that $200 \AA \mathrm{film}$ (holmium dihydride) is a better electrical conductor than the parent metal, which is similar to the behaviour of gadolinium dihydride films (Khan 1976) and holmium dihydride bulk materials (Heckman 1969). In the bulk Ho-H systems, the f.c.c. dihydride phase exists in the composition range $\mathrm{H} / \mathrm{Ho}$ (atomic ratio) $=1.95-2.24$ (Libowitz 1965). Structural investigations by Khan and Miller (1979) indicated that the films in the thickness range $70-200 \AA$ are f.c.c. dihydrides and the thickness of $200 \AA$ marks the boundary between single phase f.c.c. dihydride and mixed phase (f.c.c. dihydride +h.c.p. metal) and has the minimum resistivity value $(60 \mu \Omega-\mathrm{cm})$, which agrees with the resistivity behaviour of bulk holmium dihydride (Heckman 1969).

It may be seen from figure 1 that the resistivity of films in the thickness range $200-900 \mathrm{~A}$ (which have mixed phase) is higher than $60 \mu \Omega-\mathrm{cm}$, which is again in agreement with the bulk $\mathrm{Ho}-\mathrm{H}$ system. The increase in resistivity with the decrease of thickness in the range $70-200 \AA$ (which has a continuous structure) is due to the nonstoichiometric composition of holmium dihydride which may exist over the above thickness range. The sharp rise in resistivity below $70 \AA$ thickness is due to the discontinuous structure of the film. As the thickness of the film was increased to $900 \AA$ (which is almost h.c.p. Ho) the resistivity attained nearly a constant value $(93 \mu \Omega-\mathrm{cm})$. This value is consistent with the known bulk resistivity $(81 \mu \Omega-\mathrm{cm})$. The somewhat higher values $\left(15^{\circ}\right)$ of resistivity for the thicker films compared with the bulk metal can be attributed to inherent film defects such as point defects, vacancies, grain boundaries, dislocations etc. The resistivity of the films in the thickness range $70-99 \AA$ at $77^{\circ} \mathrm{K}$ is shown by the dashed curve (figure 1). The lower values of the resistivity at liquid nitrogen temperature are generally expected (Gillam et al 1955) and characterizes the metallic nature of the films.

The variation of resistance with temperature was measured for five films of thickness range 140 to $900 \AA$. Typical data are shown in figure 2 , in which the resistivity of the films has been plotted as a function of temperature for heating rate 2 $3 \mathrm{~K} / \mathrm{min}$. The resistivity was measured over the temperature range of $293-573^{\circ} \mathrm{K}$ at intervals of $50^{\circ} \mathrm{K}$. The increase in resistivity with temperature was found to be linear for films thicker than $140 \AA$.

The general behaviour of the temperature dependence of resistivity indicates the conduction as metallic (Chaudhury and $\mathrm{Pal}$ 1975). The temperature coefficient of 


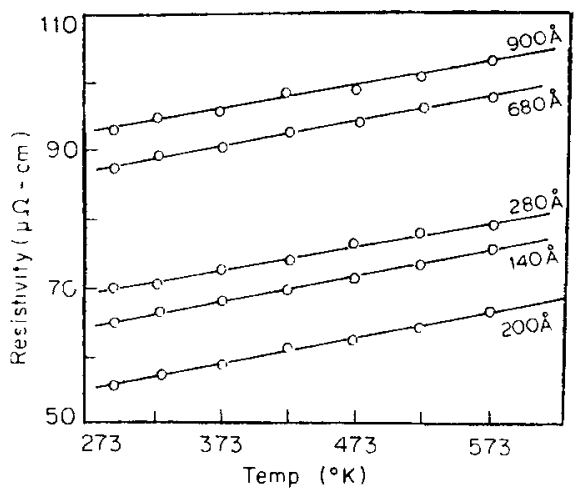

Figure 2. Variation of resistivity with temperature of holmium hydride films.

Table 1. Temperature coefficient of resistance (TCR) values of holmium hydride films.

\begin{tabular}{ll}
\hline $\begin{array}{l}\text { Film } \\
\text { thickness }(\AA)\end{array}$ & TCR $\left({ }^{\circ} \mathrm{K}\right)$ \\
\hline 140 & 0.00019 \\
200 & 0.00020 \\
280 & 0.00022 \\
680 & 0.00020 \\
900 & 0.00016 \\
\hline
\end{tabular}

resistance (TCR) of the films was measured in the range $273-343^{\circ} \mathrm{K}$ by thermally cycling the films to allow for small changes in their electrical characteristics. The observed TCR values are recorded in table 1 . There is no TCR data available for comparison (even on bulk material).

The curious feature of the results is that the resistivity of the films of limiting thickness $(200 \AA)$ is less than that of the bulk parent metal. The anomalous resistivity behaviour can be explained quite satisfactorily on the basis of the nonstoichiometric composition and consequently on the thickness dependence of the structural phase of dihydride films. The composition of the films could not be given due to experimental problems but could be well inferred from the structural investigations (Khan and Miller 1979; Khan 1976) where the diffraction pattern clearly shows that the intensities of the different reflections varied over the thickness range $200900 \AA$. The resistivity of the bulk rare earth dihydride is highly dependent on nonstoichiometric composition (Heckman 1969; Colvin et al 1960) and it is evident from the present experimental results that the resistivity depends on the structural phase (which is again dependent on thickness). The resistivity results are quite in agreement with terbium hydride films (Khan 1977) and with the behaviour of the bulk rare earth dihydride resistivity (Heckman 1969; Colvin et al 1960).

\section{Conclusion}

The effective thickness dependence of the resistivity of the holmium hydride films can be assumed to arise from a superposition of several factors, namely, the usual surface 
scattering component, structural phase change, nonstoichiometric composition of the dihydride phase and the coexistence of the dihydride and metallic phase in certain thickness range. The temperature dependence of resistivity and TCR values indicate, generally, metallic conduction characteristics.

\section{References}

Bos W G and Gayer K H 1966 J. Nucl. Mater. 181

Chopra K L 1969 Thin film phenomena. (New York; McGraw Hill)

Choudhury S and Pal A K 1975 J. Phy's. D8 1311

Colvin R V, Legvold S and Spedding F H 1960 Phys. Rev. 120741

Curzon A E and Chlebeck H G 1972 J. Less Common Metals 37411

Curzon A E and Singh O 1975 J. Less Common Metals 39227

Gasgnier M, Ghys J. Schiffmacher G, Henry C and Caro P E 1974 J. Less Common Metals 34131

Gillam E J, Preston J S and Williams B 1955 Philos. Mag. 461051

Heckman R C 1969 Sandia Laboratories SC-RR-69-571

Khan M S R 1976 Growth and structure of epitaxial rare earth hydride, Ph.D. Thesis, University of London

Khan M S R 1977 J. Bangladesh Acad. Sct. 11

Khan M S R and Miller R F 1979 J. Phys. D12 271

Kubota Y and Wallace W E 1963 J. Chem. Phys. 391285

Libowitz G G 1965 Solid state chemistry of binary metal hydrides (New York: Benjamin Inc.)

Libowitz G G 1968 Metal hydrides (New York; Academic Press)

Maissel L I and Glang R 1970 Handbook of thin film technology (New York: McGraw Hill)

Samble J R 1983 Thin Solid Films 106321

Vander Pauw 1958 Philips Res. Rep. 131 